\title{
Sorgen um die Erziehung der Kinder
}

Kinder, deren Eltern an Krebs erkrankt sind, haben ein erhöhtes Risiko für psychosoziale Probleme. Und viele krebskranke Eltern haben Bedenken, dass sie ihre elterliche Funktion nicht ausreichend wahrnehmen können. Wie es tat-

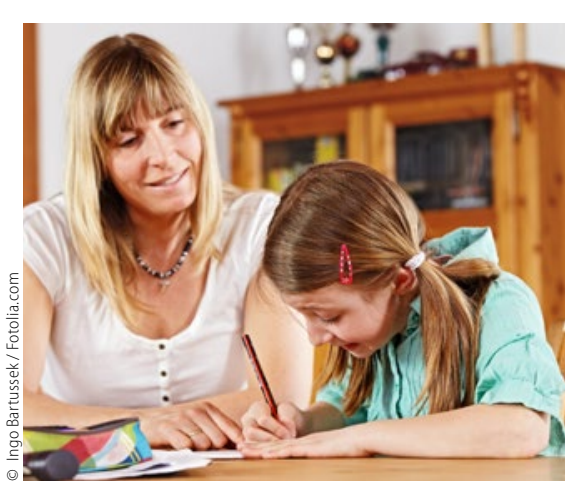

Nicht immer können krebskranke Eltern ihre Kindern bei den Hausaufgaben unterstützen. sächlich um die Fähigkeit krebskranker Eltern zur Erziehung ihrer Kinder und um die Belastung der Kinder steht, wurde nun in einer Studie untersucht. 194 erwachsene ambulante Krebspatienten mit Kindern bis zu einem Alter von 18 Jahren füllten Fragebögen zu ihrer gesundheitsbezogenen Lebensqualität, Depressionen und Angstsymptomen, zur Gesamtbelastung sowie zu ihren Ansichten und Bedenken hinsichtlich ihrer elterlichen Kompetenz aus.

$60 \%$ der befragten Patienten waren der Ansicht, dass sie die Bedürfnisse der Kinder vor ihrer Krebsdiagnose extrem gut erfüllten, aber nur $11 \%$ gaben an, dass dies auch nach der Krebsdiagnose noch der Fall sei. Die mittleren Scores für den Glauben an ihre elterliche Kompetenz sanken nach der Diagnose signifikant. Diese Abnahme korrelierte mit zunehmender Anzahl an Klinikaufenthalten, der Behandlung mit einer intravenösen Chemotherapie in den letzten Monaten, einer schlechteren gesundheitsbezogenen Lebensqualität und der Zunahme von Depressionen und Gesamtbelastung.

Bei denjenigen Patienten, die die meisten Bedenken bezüglich des Einflusses ihrer Krebserkrankung auf die Stimmung, körperliche Einschränkungen und Veränderungen im Tagesablauf ihrer Kinder hatten, war der Glauben an ihre Kompetenz als Eltern und an die Kompetenz des anderen Elternteils am stärksten reduziert. Darüber hinaus hatten diejenigen Patienten mit dem größten Vertrauensverlust in ihre elterliche Kompetenz die größten Bedenken hinsichtlich psychischer Belastungen ihrer Kinder durch die Krebserkrankung.

Diese Studie weist darauf hin, wie wichtig es ist, die Bedenken von krebskranken Eltern bezüglich ihrer Erziehungskompetenz anzusprechen.

Judith Neumaier

Moore CW et al. Parenting Changes in Adults With Cancer. Cancer. 2015;121(19):3551-7.

\section{Multiples Myelom}

\section{Antikörper mit dualem Wirkmechanismus}

Standardmäßig erfolgt die Behandlung von therapierefraktären oder rezidivierten Patienten mit multiplem Myelom mit der Kombination aus dem Immunmodulator Lenalidomid und Dexamethason. Für erfolglos vorbehandelte Patienten kommen zunehmend Dreierkombinationen zum Einsatz, allerdings limitiert aufgrund inakzeptabler Toxizität. Erstrebenswert ist daher die Kombination traditioneller Therapien mit neuen potenten Agenzien und einem günstigeren Nebenwirkungsprofil.

Einer aktuellen Studie zufolge reduzierte die Therapieerweiterung um Elotuzumab bei Patienten mit einem rezidivierten oder therapierefraktären multiplen Myelom das Risiko für ein Fortschreiten der Erkrankung oder Tod um 30 \% im Vergleich zur Standardtherapie mit Lenalidomid und Dexamethason.

Der humanisierte monoklonale Antikörper Elotuzumab ist gegen das Signaling Lymphocytic Activation Molecule F7 (SLAMF7) gerichtet, ein Glykoprotein, das auf Myelom- und natürlichen Killerzellen überexprimiert ist, nicht jedoch auf normalen Gewebezellen. Dieser erste Antikörper seiner Klasse aktiviert durch Bindung an SLAMF7 direkt natürliche Killerzellen und wirkt zellvermittelt $\mathrm{zy}$ totoxisch ohne proliferativen Effekt auf die Myelomzellen.

In der Phase-III-Studie ELOQUENT-2 verglichen die Autoren die Sicherheit und Wirksamkeit der Dreierkombination Elotuzumab, Lenalidomid und Dexamethason $(\mathrm{n}=321)$ mit der Standardtherapie (Lenalidomid und Dexamethason) ohne den Antikörper ( $\mathrm{n}=325)$. Endpunkte waren das progressionsfreie Überleben und das Gesamtansprechen.

Den Ergebnissen einer geplanten Interimsanalyse zufolge betrug das progressionsfreie Überleben in der ElotuzumabGruppe nach einem Jahr $68 \%$ gegenüber $57 \%$ im Standardtherapie-Arm, nach zwei Jahren entsprechend $41 \%$ gegenüber $27 \%$. Das mediane progressionsfreie Überleben in der Verum- im Vergleich zur Kontrollgruppe betrug 19,4vs. 14,9 Monate. Die Vorteile der Dreierkombination spiegelten sich auch im Gesamtansprechen wider: $79 \%$ vs. $66 \%$.

Insgesamt erwies sich der Antikörper als gut verträglich. Wolfgang Zimmermann

Lonial S et al. Elotuzumab therapy for relapsed or refractory myeloma. New Eng J Med. 2015;373(7): 621-31.

\section{kurz notiert}

Curt Meyer-Gedächtnispreis ausgeschrieben

Die Berliner Krebsgesellschaft e. V. stiftet den "Curt Meyer-Gedächtnispreis" in Höhe von 10.000 EUR für herausragende wissenschaftliche Leistungen in der Onkologie. Es können Publikationen, die sich mit klinischen, experimentellen oder translationalen Forschungsthemen aus dem Bereich der Onkologie befassen und die in einer Berliner Institution erarbeitet wurden, eingereicht werden. Einsendeschluss ist der 31. März 2016. Weitere Informationen: Berliner Krebsgesellschaft e. V., Prof. Dr. med. Petra Feyer, Tel.: 030 / 283 2400; E-Mail: info@ berliner-krebsgesellschaft.de. red. 